\title{
Effects of noise on hysteresis and resonance width in graphene and nanotubes resonators
}

\author{
O. G. Cantu Ros, ${ }^{1,2,3}$ G. Platero, ${ }^{3}$ and L. L. Bonilla ${ }^{1,2}$ \\ ${ }^{1}$ G. Millán Institute, Fluid Dynamics, Nanoscience and Industrial Mathematics, Universidad Carlos III de Madrid, \\ Avenida Universidad 30, E-28911 Leganés, Spain \\ ${ }^{2}$ Unidad Asociada al Instituto de Ciencia de Materiales de Madrid, CSIC, 28049 Cantoblanco, Madrid, Spain \\ ${ }^{3}$ Instituto de Ciencia de Materiales de Madrid, CSIC, 28049 Cantoblanco, Madrid, Spain
}

(Received 30 July 2012; published 21 June 2013)

\begin{abstract}
We investigate the role that noise plays in the hysteretic dynamics of a suspended nanotube or a graphene sheet subject to an oscillating force. We find that not only the size but also the position of the hysteresis region in these systems can be controlled by noise. We also find that nanoresonators act as noise rectifiers: By increasing the noise in the setup, the resonance width of the characteristic peak in these systems is reduced and, as a result, the quality factor is increased.
\end{abstract}

DOI: 10.1103/PhysRevB.87.235424

PACS number(s): 85.85.+j, 05.40.Ca, 62.25.-g

\section{INTRODUCTION}

Recent theoretical ${ }^{1,2}$ and experimental work $^{3-8}$ has shown that reducing the dimension and the size of a material with a vibrational degree of freedom enhances the role of the nonlinearities in the dynamics. Theory and experiments have demonstrated noise squeezing ${ }^{3}$ and stochastic resonance $e^{4,5,9}$ in nanomechanical resonators. These systems are used as precision measurement devices for applications such as mass and force sensing, ${ }^{10}$ quantum motion detection, and radio frequency signal processing. ${ }^{11}$ Resonators consisting of suspended nanotubes or graphene sheets can be used as extremely accurate mass sensors with yoctogram resolution. ${ }^{12}$ Models based on driven Duffing oscillators with nonlinear dissipation are used to interpret measurements in these devices. ${ }^{7,8}$ Nonlinear terms in the model are responsible for bistability in the oscillatory response as a function of the driving frequency and of quality factors dependent on the driving force.

Motivated by experiments on nanotube and graphene resonators, $7,8,12$ we study in this work the dynamics of graphene and nanotube resonators based on a suspended wire subject to an external oscillating driving force. We take into account the nonlinear damping term, the Duffing nonlinearity, and we add white noise forcing not considered in the model used to interpret the experimental results. Given the use of these resonators as accurate mass sensing devices, it is important to include in the dynamics all major effects in order to describe more accurately both the system characteristics and the different quantities inferred from them, such as mass, quality factor, dissipation coefficients, etc.

Noise is always present in a physical system. For linear systems, noise is commonly regarded as having a destructive but relatively innocuous effect, blurring our view of a system but having no effect on the underlying processes involved. In nonlinear systems, a driving white noise term can modify drastically the deterministic dynamics. It can shift bifurcation points or induce behaviors with no deterministic counterpart, thereby affecting the dynamics and the quantities measured or inferred indirectly from the experimental measurements. Previous theoretical work showed that the main sources of noise in linear simple harmonic oscillators are thermomechanical noise, temperature fluctuations, and adsorption-desorption noise. ${ }^{13}$ The analysis of the sources of noise in nonlinear resonators is beyond the scope of this work. However we will consider two different cases. On the one hand, we shall assume that the sources are similar to those of linear resonators and discuss the general case of additive white noise, without worrying about the causes of it. On the other hand, a local nonlinear damping (dissipation) means that we may need to add an appropriate white noise force to the equations according to the fluctuation-dissipation theorem. This will result in a special type of multiplicative noise. In both cases, considering the effect of noise will allow us to estimate the values of the cubic nonlinearity with higher precision than in the noiseless case. Our results may also shed some light for other possible applications.

The rest of the paper is as follows. In Sec. II we give a general discussion of the characteristics of nonlinear resonators, focusing on the role that the nonlinear dissipation coefficient plays in the resonator dynamics. In Sec. III, we add an external white noise of fixed strength to the resonator system of Sec. II and discuss the subsequent modifications of its main characteristics. In Sec. IV, we discuss the effect produced by an internal noise satisfying the fluctuation-dissipation theorem. Finally, in Sec. V we present the main results of this work and the implications that they have in experimental measurements.

\section{THEORY}

We first discuss the properties of mechanical resonators based on a suspended nanowire or graphene strip that is doubly clamped and subjected to an oscillating external force with frequency $f$. Since we only care about the dynamics of the fundamental mode, we can consider the graphene membrane as a one-dimensional object. In this regime the only difference between the nanowire and the graphene strip is given by the parameter values of each one. ${ }^{8}$

As has already been shown experimentally, ${ }^{8}$ the dynamics of these resonators is highly nonlinear, as they present a forcedependent quality factor and, in some cases, hysteresis in the oscillation amplitude as a function of the driving frequency. The resonator dynamics can be described by the following equation of motion: ${ }^{14}$

$$
m \ddot{\tilde{x}}=-k \tilde{x}-\alpha \tilde{x}^{3}-\gamma \dot{\tilde{x}}-\tilde{\eta} \tilde{x}^{2} \dot{\tilde{x}}+F_{\text {drive }} \cos (2 \pi f \tilde{t}),
$$


where $m$ is the effective mass, $\alpha x^{3}$ is the Duffing term, $\gamma \dot{\tilde{x}}$ and $\tilde{\eta} \tilde{x}^{2} \dot{\tilde{x}}$ are the linear and nonlinear damping terms, and $\left(k+\alpha x^{2}\right)$ is the amplitude-dependent spring's stiffness with a spring constant $k=m \omega_{0}^{2}\left(\omega_{0} \approx 2 \pi f\right.$ so that the drive frequency is close to resonance with the resonator natural frequency). Depending on the sign of $\alpha$, the Duffing term modifies the stiffness of the resonator (more stiff for $\alpha>$ 0 , softer for $\alpha<0)$. Equivalently, we can work with the nondimensional equation

$$
\ddot{x}=-x-Q^{-1} \dot{x}-x^{3}-\eta x^{2} \dot{x}+F_{D} \cos (\Omega t)
$$

with

$$
\begin{aligned}
x & =\tilde{x} \sqrt{\alpha m \omega_{0}^{2}}, \quad t=\omega_{0} \tilde{t}, \quad Q^{-1}=\frac{\gamma}{m \omega_{0}}, \quad \eta=\frac{\tilde{\eta} \omega_{0}}{\alpha}, \\
F_{D} & =\frac{F_{\text {drive }}}{\omega_{0}^{3}} \sqrt{\frac{\alpha}{m^{3}}}, \quad \Omega=\frac{2 \pi f}{\omega_{0}},
\end{aligned}
$$

which will be used all throughout the paper. This means that all the quantities appearing in this work are dimensionless except when specified otherwise.

Duffing resonators present a characteristic resonant line shape. They have a resonant peak for the maximum oscillation amplitude $|x|$ at the driving angular frequency $\Omega=\Omega_{\text {res }}$ and a force-dependent resonance width $\Delta \Omega=2 \pi$ FWHM (FWHM is the width at which the profile curve reaches half of its maximum value, measured from the positive minima value it reaches) which is constant (non-force-dependent) for linear resonators. When the changes to the resonance due to dissipation or noise are smaller than the resonance width obtained without consideration of these effects, the resonance is mainly due to nonlinearity and the standard definition of the quality factor is $Q=\omega_{0} \tilde{E} /\langle d \tilde{E} / d \tilde{t}\rangle=1.09 \Omega / \Delta \Omega(\tilde{E}$ is the mechanical energy at a given time and $\langle\cdots\rangle$ denotes time averaging over a time scale long compared with the oscillation period but sufficiently short that the decay of the amplitude is negligible; see the Supplemental Material in Ref. 8). For linear resonators the resonant frequency $\Omega_{\text {res }}$ and the quality factor $Q$ are independent of the driving force, ${ }^{8}$ whereas for nonlinear resonators the energy dissipation and hence the quality factor depend on the oscillation amplitude. Numerical simulations show that the resonant frequency $\Omega_{\text {res }}$ decreases with increasing $\eta$ [Fig. 1(a)] and increases with $F_{D}$ [Fig. 1(b)]. The width of the characteristic peak also depends on $\eta$ and $F_{D}$.

The dependence of $Q$ on the driving force and on the nonlinear dissipation term is not the only characteristic of Duffing resonators. One important feature of these systems is the presence of hysteresis in the oscillation amplitude as a function of the driving frequency or the driving force. ${ }^{6}$ Figure 1 shows that the system has more than one possible oscillation amplitude for a range of values of $\Omega$. In the bistable region there are two stable fixed points corresponding to periodic orbits in the case of periodically driven wires, one of larger oscillation amplitude (higher in energy) and one of smaller oscillation amplitude (lower energy). There is also one unstable periodic orbit located between the stable ones.

The two key elements in the hysteretic behavior, discussed so far in both theory and experiments, are the Duffing term, responsible for the system bistability, and the nonlinear dissipation term $\tilde{\eta} \tilde{x}^{2} \dot{\tilde{x}}$, which controls the size of the hysteresis region. In the limit of weak linear damping, perturbation theory

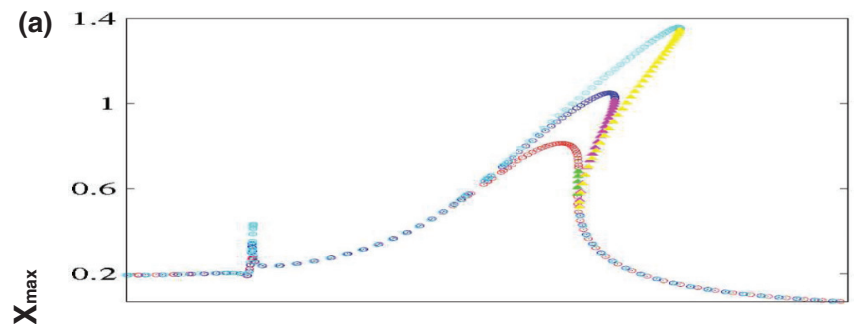

(b)

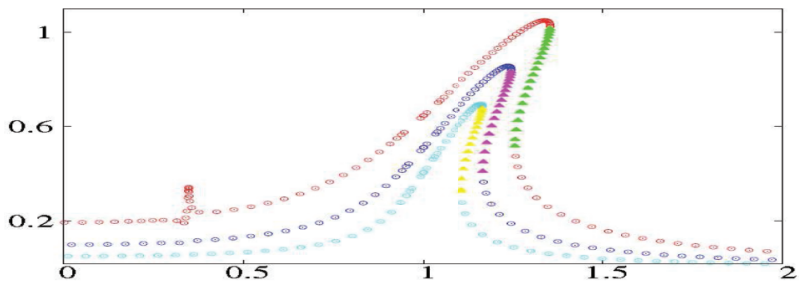

$\Omega$

FIG. 1. (Color online) Maximum oscillation amplitude, $|x|$, as a function of $\Omega$ in the case of zero noise: (a) for a fixed value of the driving force $F_{D}=0.2$ and different values of $\eta$, from bottom to top $\eta=1.2, \eta=0.5, \eta=0.2$; (b) for a fixed value of the nonlinear damping coefficient $\eta=0.5$ and different values of $F_{D}$ from bottom to top $F_{D}=0.05, F_{D}=0.1, F_{D}=0.2$. Empty circles correspond to stable orbits and solid triangles to unstable orbits. The small peak found near $\Omega \sim 1 / 3$ corresponds to a subharmonic resonance (Ref. 15). This subharmonic resonance is also observed in Fig. 3.

based on the small parameter $\epsilon=Q^{-1} \ll 1$ shows that it is necessary to have $\eta<\sqrt{3}$ for the solutions of Eq. (2) to exhibit bistability. ${ }^{1}$ However this condition only provides an upper bound. Numerical simulations show that the maximum $\eta$ for which bistability is present, $\eta_{M}$, depends on the driving force amplitude $F_{D}$. Figures 2(a) and 2(b) depict $\eta_{M}$ as a function of $F_{D}$ and the size of the hysteresis window (the range of frequencies at which there is hysteresis), $\Delta H$, as a function of $\eta$. Note that $\eta_{M}=1.325$ at $F_{D}=0.2$, indicated in Fig. 2(a), is appreciably smaller than the upper bound $\sqrt{3}$, whereas for a much smaller $F_{D}=3.8 \times 10^{-3}, \eta_{M}=1.642$ as indicated in Fig. 2(b) is closer to $\sqrt{3}$. Increasing $\eta$ reduces the frequency range at which the system is bistable. For a given driving force $F_{D}$, the minimum frequency $\Omega_{\min }$ at which there is bistability does not vary too much, whereas the maximum frequency $\Omega_{\max }$ at which hysteresis can be observed moves toward smaller values. In Fig. 2(a), $\Omega_{\min }=1.257$ and $\Omega_{\max }$ is seemingly unbounded for $\eta=0$. For $\eta=0.5, \Omega_{\text {min }}$ has moved slightly to $\Omega_{\min }=1.251$ while the maximum frequency has been considerably reduced to $\Omega_{\max }=1.357$. This reduction continues until $\eta$ reaches a critical point where $\Omega_{\min }=\Omega_{\max }$ and bistability completely disappears. This happens at $\eta=$ 1.325 in the case of $F_{D}=0.2$ [Fig. 2(a)], and at $\eta=1.642$ for $F_{D}=3.8 \times 10^{-3}$ [Fig. 2(b)]. Eichler et al. estimate $\tilde{\eta}=$ $7.9 \times 10^{5} \mathrm{~kg} /\left(\mathrm{m}^{2} \mathrm{~s}\right)>\alpha \sqrt{3} / \omega_{0}$ (corresponding to $\left.\eta>\sqrt{3}\right)$ for carbon nanotubes and $\tilde{\eta}=1.5 \times 10^{5} \mathrm{~kg} /\left(\mathrm{m}^{2} \mathrm{~s}\right)<\alpha \sqrt{3} / \omega_{0}$ $(\eta<\sqrt{3})$ for graphene resonators. ${ }^{8}$

So far the only parameter controlling the size of the hysteresis region or the dependence of $Q$ on the driving force has been the nonlinear dissipation coefficient $\eta$. This coefficient is an intrinsic property of each material and is fixed for every setup. However there is a parameter that may give rise to similar effects and that can be tuned or controlled externally. 


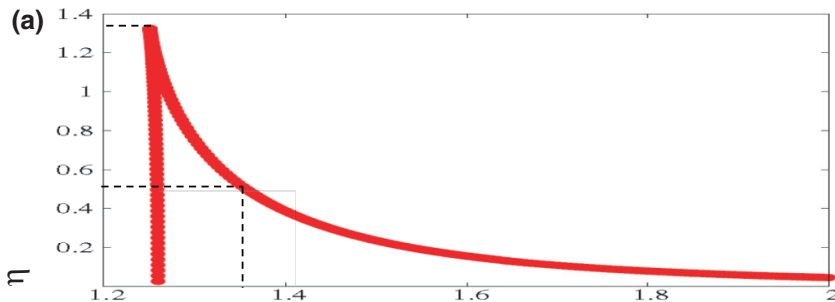

(b)

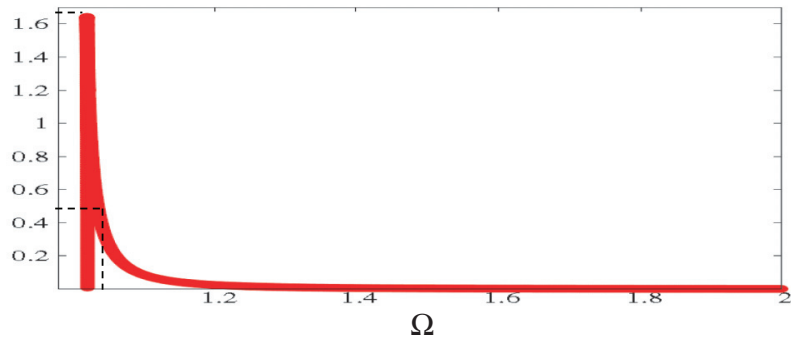

FIG. 2. (Color online) Bistability zone as function of nonlinear dissipation amplitude $\eta$ and $\Omega=2 \pi f$ for fixed values of the driving amplitude: (a) $F_{D}=0.2$ and (b) $F_{D}=3.8 \times 10^{-3}$. The projection of the red curve on the $\Omega$ axis represents the minimum and maximum values of $\Omega$ at which the system is bistable. The size of the hysteresis window, $\Delta H=\Omega_{\max }-\Omega_{\min }$, depends on both the driving force and the nonlinear dissipation parameter [compare panels (a) and (b)]. For example, for $\eta=0.5$, the minimum (maximum) frequency at which hysteresis can be observed is $\Omega_{\min }=1.262\left(\Omega_{\max }=1.357\right)$ in panel (a) and $\Omega_{\min }=1.02\left(\Omega_{\max }=1.034\right)$ in panel (b). As $\eta$ increases, $\Delta H$ gets reduced until the inflection point, $\Omega_{\max }=\Omega_{\min }$, at which hysteresis completely disappears.

In the next section we explore the effect that the addition of noise has on the dynamics of the system and show that it has properties similar to those found for $\eta$.

\section{EXTERNAL NOISE: RESULTS AND DISCUSSION}

According to the fluctuation-dissipation theorem, noise accompanies the process of energy dissipation. Thus we can expect behaviors similar to those related to $\eta$ when noise is taken into account. For such purpose we add an external white noise to Eq. (2)

$$
\begin{aligned}
\ddot{x} & =-x-Q^{-1} \dot{x}-x^{3}-\eta x^{2} \dot{x}+F_{D} \cos (\Omega t)+\sigma \xi(t), \\
\langle\xi(t)\rangle & =0, \quad\left\langle\xi(t) \xi\left(t^{\prime}\right)\right\rangle=\delta\left(t-t^{\prime}\right),
\end{aligned}
$$

which is presumably larger than the intrinsic noise. Numerical simulation of this stochastic equation shows that the noise shrinks the hysteresis window [Figs. 3, 4(a), and 4(b)] and reduces the resonance width (Fig. 5).

For fixed values of $F_{D}$ and $\eta$, the hysteresis window is reduced by increasing the noise strength, $\sigma$. Figure 3 shows that at $\eta=0.5$, the hysteresis window has been reduced from $\Delta H=0.098$ at $\sigma=0$ to $\Delta H=0.04$ at $\sigma=0.014$. In contrast with the effect of increasing $\eta$, increasing $\sigma$ builds up rapidly the minimum frequency $\Omega_{\min }$ at which bistability starts, whereas the maximum frequency $\Omega_{\max }$ is slightly above than that of the deterministic case but it does not change that much with $\sigma$; see Fig. 4. Let us now compare the effects of $\eta$ and $\sigma$ on the hysteresis window. In Fig. 2, we decrease the window size by increasing $\eta$ from zero to $\eta=0.7671$ at zero noise, whereas in Fig. 4(a) we do so by increasing $\sigma$ from zero

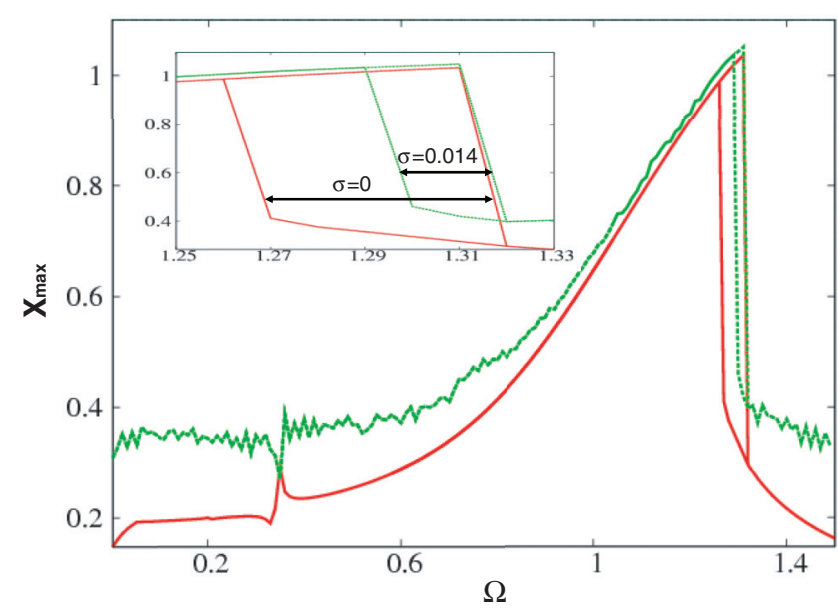

FIG. 3. (Color online) Curve of the maximum oscillation amplitude as a function of $\Omega$ at zero noise (solid line) and at $\sigma=0.014$ (doted line) for a given $\eta=0.5, F_{D}=0.2, Q^{-1}=5 \times 10^{-4}$. The area enclosed by the solid square corresponds to the bistability zone at zero noise, and the area enclosed by the dotted square represents the bistability zone for a finite noise amplitude $\sigma$. Inset: Zoom of the bistability region.

to $\sigma=0.014$ at $\eta=0.5$. In both cases $\Delta H=0.04$ is the same; however $\Omega_{\max }$ and $\Omega_{\min }$ are different for the same $F_{D}=0.2$ : (i) $\Omega_{\max }=1.299, \Omega_{\min }=1.259$ for $\eta=0.7671$ and $\sigma=0$, and (ii) $\Omega_{\max }=1.318, \Omega_{\min }=1.278$ for $\sigma=0.014$ and $\eta=0.5$. The hysteresis window has shifted towards higher values of $\Omega$. The critical value at which hysteresis disappears, $\Omega_{\max }=\Omega_{\min }$, occurs at $\Omega=1.251$ for $\sigma=0$ and $\eta=1.325$ [Fig. 2(a)]. However, for $\eta=0.5$ and $\sigma=0.014$, it occurs at $\Omega=1.369$, above the original zone of hysteresis at zero noise [shaded area in Fig. 4(a)]. Summarizing, increasing $\sigma$
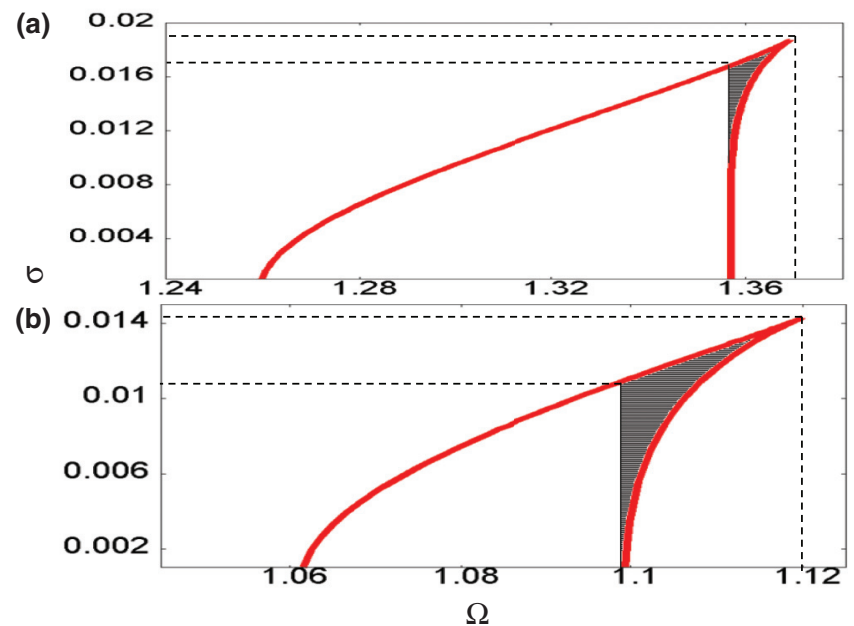

FIG. 4. (Color online) Bistability zone in the plane $(\sigma, \Omega)$ for fixed nonlinear damping $\eta=0.5$ and (a) $F_{D}=0.2$, (b) $F_{D}=0.02$. The projection of the curve on the $\Omega$ axis gives the minimum and maximum frequencies at which there is hysteresis. The hysteresis region gets smaller as the noise is increased. Comparing (a) and (b), we can observe that this region also gets smaller for smaller values of FD. The shaded zone corresponds to values of $\Omega$ at which there is no hysteresis for $\sigma=0$. 

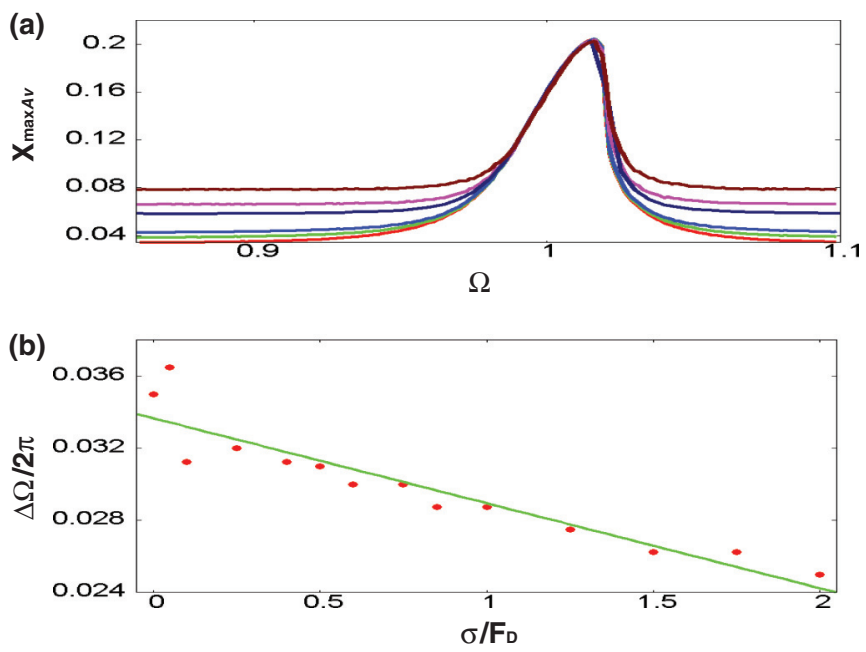

FIG. 5. (Color online) (a) Averaged maximum oscillation amplitude $X_{\operatorname{maxAv}}$ as a function of $\Omega$ for $\eta=1.6$ and $F_{D}=0.0038$ and different values of $\sigma$, from bottom to top $\sigma=0.4 F_{D}, \sigma=0.5 F_{D}$, $\sigma=0.6 F_{D}, \sigma=F_{D}, \sigma=1.25 F_{D}, \sigma=1.5 F_{D}$. (b) $\Delta \Omega / 2 \pi$ (width at the half-maximum value) as a function of $\sigma$ for the profile curves in (a). Dots correspond to numerical results and solid line corresponds to a linear fitting with $\Delta \Omega / 2 \pi=0.033669-0.0047149 \sigma$.

not only reduces the hysteresis window (as has already been found experimentally); ${ }^{10}$ it also shifts the window towards higher frequencies. This is so to the point that, just before the critical frequency $\Omega_{\max }=\Omega_{\min }$, hysteresis is found in a frequency region in which there is no bistability at zero noise [shaded area in Figs. 4(a) and 4(b)].

The fact that the hysteresis window moves towards higher frequency values when $\sigma$ increases is more than just a curiosity. It is telling us a lot about what is going on in the system: It means that noise is feeding energy into the system. This can be understood with the following argument. In the hysteretic region there are three periodic orbits; one unstable orbit separates stable orbits with larger and smaller amplitude (therefore with higher and lower energy), as seen in Figs. 1 and 3. When the hysteresis zone is reduced, only one of the stable orbits survives. The other stable orbit coalesces with the unstable one and both then disappear. As $\eta$ increases the surviving orbit has the smaller amplitude: As $\eta$ increases, the system is dissipating more energy and its dynamics selects lower energy orbits. In contrast to this, the larger amplitude orbit survives when $\sigma$ increases because the noise is feeding energy to the system making it more likely for the system to oscillate around the higher energy periodic orbit. It also turns out that the quality factor increases with noise which enforces this argument.

Even more important than the reduction of $\Delta H$ is that increasing the noise strength makes the resonance width, $\Delta \Omega$, thinner for a large range of values of $\sigma$. The effect that resonance width shrinks with noise is always present, even in systems not exhibiting hysteresis. In Fig. 5(a) the change of the profile curve with $\sigma$ is plotted. In this figure the average value of the maximum oscillation amplitudes reached over a long period of time is plotted. Figure 5(b) shows the resonance width as a function of $\sigma$ for the averaged maximum amplitude profile curve. Figure 5 shows that the resonance width decreases with $\sigma$.

The modification of the resonance width indicates that the presence of noise is affecting the value of the quality factor $Q$. Since the reductions/enlargements of the resonant width due to noise and dissipation are small compared with the zero-noise resonance width, the quality factor is ${ }^{8}$

$$
Q=\Omega_{0} \frac{\langle E\rangle}{\langle\dot{E}\rangle} \approx 1.09 \frac{\Omega}{\Delta \Omega},
$$

where $E$ is the mechanical energy at a given time $t$. According to the Supplemental Material in Ref. 8, the approximation $Q \approx 1.09 \Omega / \Delta \Omega$ holds in the limit of very large quality factor. The noise reduces $\Delta \Omega$ and therefore it enhances the quality factor. This contrasts with the behavior of linear systems. In them, external noise increases energy dissipation and makes these systems less efficient. However for our nonlinear system, the random energy provided by noise gets absorbed by the system and converted in useful mechanical energy, thereby yielding a higher quality factor. In this sense, the nonlinearly damped driven Duffing oscillator acts in average on time as a noise rectifier that improves its performance by using energy provided by the external noise.

The reduction of the frequency width is also telling us that the value of $\eta$ calculated from the experiments (in which noise is certainly present) may be smaller than the real one; i.e., $\left.\eta\right|_{\sigma=0}>\left.\eta\right|_{\sigma>0}$. The value of $\eta$ in Ref. 8 is calculated from the expression

$$
\eta=\left(\frac{m \Delta f}{0.032}\right)^{3}\left(\frac{F_{D}}{f_{0}}\right)^{2},
$$

where the dependence of $\Delta \Omega$ on $\sigma$ has not been taken into account. We can include this dependence in Eq. (5) and let $\eta(\sigma)$ be given by (5) for a fixed value of $\sigma \geqslant 0$. We can rewrite $\Delta \Omega=\left.\Delta \Omega\right|_{\sigma=0} / f(\sigma)$, with $f(\sigma)=\left(\left.\Delta \Omega\right|_{\sigma=0} /\left.\Delta \Omega\right|_{\sigma>0}\right)$. The factor $f(\sigma)$ indicates how the resonance width changes with noise: $f(0)=1$ and $f(\sigma)>1$ for $\sigma>0$. Using (5), we see that

$$
\eta(\sigma)=\frac{\eta(0)}{f(\sigma)^{3}} \leqslant \eta(0) .
$$

Thus the value $\eta(\sigma)$ is screened by the effect of noise.

\section{INTERNAL NOISE}

So far we have discussed a general case of constantamplitude external white noise without specifying its nature. However, in our system there are different sources of dissipation that contribute to $\gamma$ and $\tilde{\eta}$ in (1). Let us assume that the dominant noise is thermomechanical. ${ }^{17}$ According to the fluctuation-dissipation theorem, there should be a stochastic white noise forcing term in (3) with an appropriate strength, $\sigma^{2}=2 \delta\left(\eta x^{2}+1 / Q\right)$, where $\delta=\alpha k_{B} T / k^{2}$. We have performed numerical simulations of (3) with this internal noise for a given value of $\eta$. We see in Fig. 6 that the obtained results are qualitatively similar to those found using external noise [Figs. 5(a) and 5(b)]: When the dominant noise is thermomechanical the resonance width decreases with $T$ for low temperatures. Figure 6(b) shows that on average increasing the temperature decreases the resonance width (similar to 

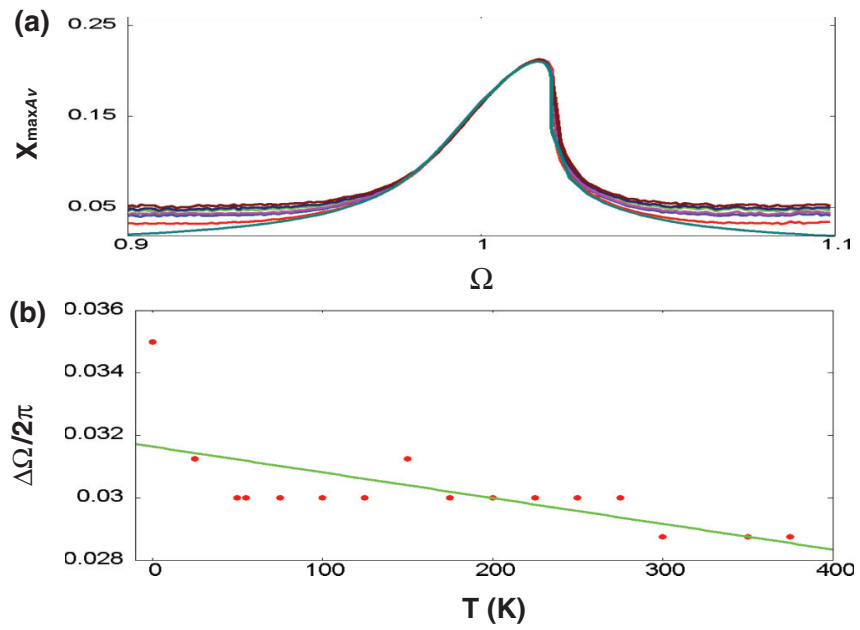

FIG. 6. (Color online) (a) Averaged maximum oscillation amplitude, $X_{\text {maxAv }}$, for $\eta=1.6$ and $F_{D}=0.0038$ as a function of $\Omega$. The noise strength is given by the fluctuation-dissipation theorem, $\sigma^{2}=2 \delta\left(\eta x^{2}+1 / Q\right)$, with $\delta=\alpha k_{B} T /\left(k^{2}\right)$. From bottom to top: $T=0, T=25 \mathrm{~K}, T=100 \mathrm{~K}, T=125 \mathrm{~K}, T=175 \mathrm{~K}, T=200 \mathrm{~K}$, $T=350 \mathrm{~K}$. (b) $\Delta \Omega / 2 \pi$ (width at the half-maximum value) as a function of $T$ for the profile curves in (a). Dots correspond to numerical results and solid line corresponds to a linear fitting with $\Delta \Omega / 2 \pi=-8.2401 \times(10)^{-6} T+0.031640$.

the effect of increasing the fixed strength $\sigma$ of an external noise), thereby increasing the quality factor. Also the hysteresis frequency range diminishes with all the consequences already mentioned.

The measurements of the quality factor made by Eichler et al. are not affected by these results, since they calculate $Q=1.09 \Omega_{0} / \Delta \Omega$ and take $\Delta \Omega$ from the experimental measurements which are affected by the existing noise. However they calculated $\tilde{\eta}$ without taking noise into account. The measurements in Ref. 8 were interpreted assuming that only the nonlinear dissipation $\tilde{\eta}$ was responsible for the shrinking or expansion of the resonant peak. Since the dissipation $\tilde{\eta}(\sigma)$ is smaller in the presence of noise, the value of $\tilde{\eta}$ proposed in Ref. 8 could be smaller than that in the real sample.

\section{CONCLUSIONS}

We have found that the presence of noise modifies in a qualitative way the dynamics of the nonlinear Duffing resonators, and it gives rise to some effects similar to those attributed to the nonlinear dissipation coefficient. Noise not only reduces the window of hysteresis but also shifts it to higher values of the driving frequency. Since the value of $\eta$ is inherent to the material and cannot be tuned, there is not much room for modifying the quality factor rather than decreasing $F_{D}$ which cannot always be done experimentally. However controlling the sources of noise allows us to increase or decrease the bistability regions and even to shift them to higher frequencies within easy experimental reach.

We have also found that the resonant width can be controlled by noise and hence the quality factor can be increased by increasing the sources of noise in the system. This means not only that higher quality factors can be reached by increasing the sources of noise, but also, and even more important, it means that nanoresonators act as noise rectifiers. This is the main result of this work, to show that nonlinear nanoresonators are able to convert random energy from the environment into useful mechanical energy.

Even though there are different sources of noise present in these systems, ${ }^{13,17}$ in this work we have discussed mostly the case of white noise with constant strength $\sigma$, leaving its nature unspecified. However, we also performed numerical simulations assuming that the dominant noise is of thermomechanical origin and satisfies the fluctuation-dissipation theorem (the noise strength $\sigma=\sqrt{2 \delta\left(\eta x^{2}+1 / Q\right)}$, with $\delta=\alpha k_{B} T / k^{2}$, depends on the temperature and on the oscillation amplitude). The results are qualitatively the same as those obtained for constant-strength white noise.

\section{ACKNOWLEDGMENTS}

This work was supported by the Spanish Ministerio de Economía y Competitividad Grants No. FIS2011-28838-C0201, No. FIS2010-22438-E (Spanish National Network Physics of Out-of-Equilibrium Systems), and No. MAT 2011-24331, and ITN, Grant No. 234970 (EU).
${ }^{1}$ R. Lifshitz and M. C. Cross, in Reviews of Nonlinear Dynamics and Complexity, edited by H. G. Schuster, Vol. I (Wiley-VCH, New York, 2008).

${ }^{2}$ J. Moser et al., in Fluctuating Nonlinear Oscillators: From Nanomechanics to Quantum Superconducting Circuits, edited by M. Dykman (Oxford University Press, Oxford, 2012), p. 341.

${ }^{3}$ R. Almog, S. Zaitsev, O. Shtempluck, and E. Buks, Phys. Rev. Lett. 98, 078103 (2007).

${ }^{4}$ R. L. Badzey and P. Mohanty, Nature (London) 437, 995 (2005).

${ }^{5}$ R. Almog, S. Zaitsev, O. Shtempluck, and E. Buks, Appl. Phys. Lett. 90, 013508 (2007).

${ }^{6}$ I. Kozinsky, H. W. Ch. Postma, O. Kogan, A. Husain, and M. L. Roukes, Phys. Rev. Lett. 99, 207201 (2007).

${ }^{7}$ J. S. Bunch, A. M. van der Zande, S. S. Verbridge, D. M. Tanenbaum, J. M. Parpia, H. G. Craighead, and P. L. McEuen, Science 315, 490 (2007).
${ }^{8}$ A. Eichler, J. Chaste, M. Zdrojek, I. Wilson-Rae, and A. Bachtold, Nat. Nanotechnol. 6, 339 (2011).

${ }^{9}$ M. I. Dykman and M. A. Krivoglaz, Sov. Phys. JETP 50, 30 (1979).

${ }^{10}$ J. S. Aldridge and A. N. Cleland, Phys. Rev. Lett. 94, 156403 (2005).

${ }^{11}$ V. A. Sazonova, Y. Yaish, H. Üstünel, D. Roundry, T. A. Arias, and P. L. McEuen, Nature (London) 431, 284 (2004); P. Poncharal, Z. L. Wang, D. Ugarte, and W. A. Heer, Science 283, 1513 (1999); B. Reulet, A. Yu. Kasumov, M. Kociak, R. Deblock, I. I. Khodos, Yu. B. Gorbatov, V. T. Volkov, C. Journet, and H. Bouchiat, Phys. Rev. Lett. 85, 2829 (2000); S. T. Purcell, P. Vincent, C. Journet, and V. T. Binh, ibid. 89, 276103 (2002); B. Witkamp, M. Poot, and H. S. J. van der Zant, Nano Lett. 6, 2904 (2006); D. Garcia-Sanchez, A. San Paulo, M. J. Esplandiu, F. Perez-Murano, L. Forró, A. Aguasca, and A. Bachtold, Phys. Rev. Lett. 99, 085501 (2007); 
B. Lassagne, D. Garcia-Sanchez, A. Aguasca, and A. Bachtold, Nano Lett. 8, 3735 (2008); K. Jensen, K. Kim, and A. Zettl, Nat. Nanotechnol. 3, 533 (2008); H.-Y. Chiu, P. Hung, H. W. C. Postma, and M. Bockrath, Nano Lett. 8, 4342 (2008); A. Eriksson, S. W. Lee, A. A. Sourab, A. Isacsson, R. Kaunisto, J. M. Kinaret, and E. E. B. Campbell, ibid. 8, 1224 (2008).

${ }^{12}$ J. Chaste, A. Eichler, J. Moser, G. Ceballos, R. Rurali, and A. Bachtold, Nat. Nanotechnol. 7, 301 (2012).

${ }^{13}$ A. N. Cleland and M. L. Roukes, J. Appl. Phys. 92, 2758 (2002).

${ }^{14}$ A. H. Nayfeh and D. T. Mook, Nonlinear Oscillations (Wiley, New York, 1995).
${ }^{15}$ Another important difference between the linear and nonlinear resonators is the existence of subharmonic resonances. In linear resonators there is only one resonant frequency when the driving force vibrates at the natural frequency of the oscillator. In nonlinear resonators, subharmonic resonant frequencies of smaller amplitude can be found. The small peak around $\Omega \sim 1 / 3$ in Fig. 1 is an example. Subharmonic resonances are beyond the scope of this paper and we will not further describe them. A more detailed explanation can be found in Ref. 16.

${ }^{16}$ L. D. Landau and E. M. Lifshitz, Mechanics, 3rd ed. (Pergamon, New York, 1976).

${ }^{17}$ V. A. Sazonova, Ph.D. thesis, Cornell University, 2006. 